\title{
Tick Thioester-Containing Proteins and Phagocytosis Do Not Affect Transmission of Borrelia afzelii from the Competent Vector Ixodes ricinus
}

\author{
Veronika Urbanová, Ondřej Hajdušek, Helena Hönig Mondeková ${ }^{+}$, Radek Šíma and \\ Petr Kopáček* \\ Biology Centre of the Czech Academy of Sciences, Institute of Parasitology, Ceske Budejovice, Czechia
}

\section{OPEN ACCESS}

Edited by:

Sarah Irène Bonnet, Institut National de la Recherche Agronomique (INRA), France

Reviewed by:

Edward Shaw,

Oklahoma State University-Stillwater,

USA

Melissa Jo Caimano,

University of Connecticut Health

Center, USA

*Correspondence: Petr Kopáček

kopajz@paru.cas.cz

${ }^{\dagger}$ Present Address:

Helena Hönig Mondeková, Institute of Microbiology, Czech Academy of Sciences, Trebon,

Czechia

Received: 28 November 2016 Accepted: 27 February 2017

Published: 16 March 2017

Citation:

Urbanová V, Hajdušek O, Hönig Mondeková H, Šima R and Kopáček $P$

(2017) Tick Thioester-Containing

Proteins and Phagocytosis Do Not Affect Transmission of Borrelia afzeli from the Competent Vector Ixodes

Front. Cell. Infect. Microbiol. 7:73. doi: 10.3389/fcimb.2017.00073
The present concept of the transmission of Lyme disease from Borrelia-infected Ixodes $\mathrm{sp}$. ticks to the naive host assumes that a low number of spirochetes that manage to penetrate the midgut epithelium migrate through the hemocoel to the salivary glands and subsequently infect the host with the aid of immunomodulatory compounds present in tick saliva. Therefore, humoral and/or cellular immune reactions within the tick hemocoel may play an important role in tick competence to act as a vector for borreliosis. To test this hypothesis we have examined complement-like reactions in the hemolymph of the hard tick Ixodes ricinus against Borrelia afzelii (the most common vector and causative agent of Lyme disease in Europe). We demonstrate that I. ricinus hemolymph does not exhibit borreliacidal effects comparable to complement-mediated lysis of bovine sera. However, after injection of $B$. afzelii into the tick hemocoel, the spirochetes were efficiently phagocytosed by tick hemocytes and this cellular defense was completely eliminated by pre-injection of latex beads. As tick thioester-containing proteins (T-TEPs) are components of the tick complement system, we performed RNAi-mediated silencing of all nine genes encoding individual T-TEPs followed by in vitro phagocytosis assays. Silencing of two molecules related to the C3 complement component (IrC3-2 and IrC3-3) significantly suppressed phagocytosis of $B$. afzelii, while knockdown of IrTep (insect type TEP) led to its stimulation. However, RNAi-mediated silencing of T-TEPs or elimination of phagocytosis by injection of latex beads in $B$. afzelii-infected $I$. ricinus nymphs had no obvious impact on the transmission of spirochetes to naïve mice, as determined by $B$. afzelii infection of murine tissues following tick infestation. This result supports the concept that Borrelia spirochetes are capable of avoiding complement-related reactions within the hemocoel of ticks competent to transmit Lyme disease.

Keywords: Borrelia, complement, Ixodes, phagocytosis, thioester-containing proteins

\section{INTRODUCTION}

Ticks are obligatory blood-feeders capable of transmitting a wide variety of pathogens including viruses, bacteria, protozoa, fungi, or nematodes to their vertebrate hosts (Jongejan and Uilenberg, 2004; De La Fuente et al., 2008). Yet, the vector competence of different tick species is quite specific and is determined by the ability of any particular pathogen to overcome several barriers on its route 
from the infected tick to the naïve host, including defense mechanisms within the tick midgut, hemocoel, and salivary glands; for review see (Hajdusek et al., 2013). One of the most thoroughly investigated tick-borne diseases of humans is Lyme disease, caused by spirochetes of the genus Borrelia (Burgdorfer et al., 1982; Stanek et al., 2012) transmitted mainly by Ixodes scapularis in the USA and Ixodes ricinus in Europe (Piesman and Gern, 2004; Radolf et al., 2012). Several tick molecules have been described to play important roles in the Borrelia transmission cycle; reviewed in Hajdusek et al. (2013) and Kung et al. (2013): Glutathione peroxidase Salp 25D facilitates spirochete acquisition from the infected host (Narasimhan et al., 2007); TROSPA, tick receptor for the outer surface protein A, plays a role in Borrelia long-term persistence within the tick gut (Pal et al., 2004); tre31 that binds to another outer Borrelia surface lipoprotein, BBE 31, allows crossing of the midgut barrier (Zhang et al., 2011). Salivary proteins Salp15 (Ramamoorthi et al., 2005), tick histamine release factors (Dai et al., 2010), and tick salivary lectin pathway inhibitor (Schuijt et al., 2011) protect Borrelia at the tick-host interface via modulation of the host immune response. In addition to tick molecules, transmission of spirochetes is also aided by proteins originating from the blood meal, such as host plasminogen that is bound and activated on the spirochete surface, facilitating Borrelia migration through the tick and dissemination in the host (Coleman et al., 1997).

A previous study suggested that the difference in capacity to transmit Borrelia burgdorferi between I. scapularis (competent tick) and Dermacentor variabilis (refractory tick) is likely to be due to higher borreliacidal and phagocytic activities in the hemolymph of D. variabilis (Johns et al., 2001). Despite this important observation, our current knowledge on tick-Borrelia interactions in the hemocoel of Ixodes sp. ticks is rather limited. Phagocytosis of B. burgdorferi by I. scapularis hemocytes upon spirochete penetration from the midgut to the hemocoel has been described in several studies (Coleman et al., 1997; DunhamEms et al., 2009) but whether this cellular defense reaction plays any role in Borrelia transmission in a competent vector remains unclear.

In vertebrate animals, including mammalian, avian or reptile hosts, the decisive role in susceptibility or resistance to infection by a certain genospecies of B. burgdorferi sensu lato complex, is most likely played by the serum complement system (Kurtenbach et al., 1998, 2002; Kuo et al., 2000; Bhide et al., 2005; De Taeye et al., 2013). A borreliacidal effect on spirochetes was reported to be exerted by the alternative pathway of mammalian complement (Kurtenbach et al., 1998; Kuo et al., 2000). Since ticks possess a primitive complement system, comprising thioester-containing proteins (TEPs), ficolin-like lectins or putative C3-convertases (Kopacek et al., 2012), we primarily asked whether or not tick complement plays a role in the competence of ticks to act as a vector for Lyme disease. In this study we focused on molecules of the TEP family, which, in Ixodes sp. ticks, involves representatives of four major classes of TEPs known in invertebrates: (i) three proteins related to C3-complement component; (ii) three different $\alpha_{2}$-macroglobulins; (iii) one insect-type TEP, and (iv) two macroglobulin-complementrelated (MCR) molecules (Buresova et al., 2011; Urbanova et al., 2015). Using RNAi-mediated silencing of individual genes encoding tick TEPs (T-TEPs), followed by in vitro phagocytosis assays, we have previously demonstrated that different T-TEPs are involved in phagocytosis of different model microbes (Gramnegative Chryseobacterium indologenes, Escherichia coli and yeast Candida albicans) by tick hemocytes (Buresova et al., 2009, 2011; Urbanova et al., 2015). However, a similar study focusing on Borrelia sp. spirochetes has not been performed yet, mainly due to the lack of a reliable phagocytic assay for this tick-borne pathogen. To overcome this problem, we have implemented a phagocytic assay for Borrelia sp. that exploits dual labeling, making it possible to clearly distinguish free and/or attached spirochetes from those being engulfed by tick hemocytes. Phagocytosis of different microbes by invertebrate hemocytes could be efficiently blocked by intra-hemocoelic injection of inert particles such as latex or polystyrene beads, as previously demonstrated for the fruit fly Drosophila melanogaster (Nehme et al., 2011) or the tick I. scapularis (Liu et al., 2011). With this experimental background, and using a recently established laboratory transmission model for B. afzelii (the most important agent of Lyme disease in Europe), we have examined the role of T-TEPs in phagocytosis of spirochetes by tick hemocytes and addressed the question of whether or not this cellular defense plays a role in spirochete transmission from Borrelia-infected I. ricinus nymphs to naïve mice. Our results collectively demonstrate that complement-like molecules are involved in tick phagocytic responses to $B$. afzelii, but do not prevent transmission of $B$. afzelii. These findings add to our understanding of the competence of this tick species to act as a vector for Lyme disease.

\section{MATERIALS AND METHODS}

\section{Biological Material}

Adult females and males of I. ricinus were collected by flagging in woodlands around České Budějovice, the Czech Republic. All developmental stages (eggs, larvae, nymphs, and adults) were maintained in wet chambers with a humidity of about $95 \%$, temperature $24^{\circ} \mathrm{C}$ and day/night period set to $15 / 9 \mathrm{~h}$. Females were fed naturally on laboratory guinea pigs. The larvae were fed on guinea pigs, allowed to molt to nymphs and, after 4-6 weeks, further fed on guinea pigs or rabbits. The nymphs (pathogen free or infected with B. afzelii $\mathrm{CB} 43$ ) and adult females (pathogen free) were used for experiments described below. All laboratory animals were treated in accordance with the Animal Protection Laws of the Czech Republic No. 246/1992 Sb., ethics approval No. 095/2012.

\section{Borreliacidal Assay}

B. afzelii CB43 spirochetes were cultivated in BSK-H complete medium (Sigma-Aldrich) at $33^{\circ} \mathrm{C}$ for 5-7 days and for the assay, were diluted to a concentration of $5 \times 10^{6}$ cells $/ \mathrm{ml}$. Hemolymph samples from 50 semi-engorged females (6th day of feeding) were collected into a glass capillary from the cut front leg, immediately cooled on ice and the collected pool was centrifuged at $300 \times$ $\mathrm{g}$ for $10 \mathrm{~min}$. The supernatant was transferred to a fresh tube and centrifuged at $9500 \times \mathrm{g}$ for $10 \mathrm{~min}$. The hemocyte-free 
plasma so obtained was used for subsequent experiments. Bovine serum was prepared from manually defibrinated bovine blood as described previously (Perner et al., 2016) and inactivated bovine serum was prepared by heating at $56^{\circ} \mathrm{C}$ for $40 \mathrm{~min}$.

The assay was performed in 96-well plates (Nunc) by adding $50 \mu \mathrm{l}$ of $B$. afzelii $\left(5 \times 10^{6} / \mathrm{ml}\right)$ to $10 \mu \mathrm{l}$ of tested sample (tick plasma, bovine serum, inactivated bovine serum, or BSK-H medium as a control), and incubated for $24 \mathrm{~h}$ in a wet chamber at $33^{\circ} \mathrm{C}$. The number of live B. afzelii was determined using darkfield microscopy as follows. The Borrelia culture $(3.5 \mu \mathrm{l})$ was transferred onto a microscope slide and covered with $18 \times 18$ $\mathrm{mm}$ coverslip. Spirochetes present in the view field were counted and the average number, calculated from 10 fields counts, was multiplied by the coefficient $3.9 \times 10^{5}$ pre-determined for our microscope Olympus, model BX53 (total magnification-400x). The obtained results thus represent the number of Borrelia normalized for $1 \mathrm{ml}$ culture volume.

\section{In vivo Phagocytosis of Borrelia afzelii CB43}

Pathogen free, adult $I$. ricinus females were fed naturally for 6 days on guinea pigs. Cultivated B. afzelii $\left(5 \times 10^{4}\right.$ spirochetes per tick) were injected into the hemocoel of semi-engorged females in a volume of $138 \mathrm{nl}$ by microinjection (microinjector Drummond). Hemolymph samples from individual ticks were collected at defined time points $(0,1,3$, and $6 \mathrm{~h})$ after injection of spirochetes and mixed with $10 \mu \mathrm{l}$ of L15-BOFES medium supplemented with $10 \%$ fetal calf serum (PAA Laboratories) on microscope slides. Cells were fixed with $4 \%$ formaldehyde in phosphate saline buffer (PBS) for $20 \mathrm{~min}$ and washed 3 times with PBS. Spirochetes were stained with primary rabbit anti-B. burgdorferi (Thermo Scientific) antibody (1:200 in PBS), on the slides and incubated on a horizontal shaker at room temperature (RT) for $1 \mathrm{~h}$. After washing with PBS (3 times/5 $\mathrm{min}$ ), slides were stained with fluorescently labeled goat antirabbit secondary antibody (Alexa 594) (Molecular Probes) diluted 1:500 in PBS and incubated for $1 \mathrm{~h}$ at RT. Hemocytes were then permeabilized using $1 \%$ Triton X-100 in PBS with $1 \%$ BSA (Bovine serum albumin, Sigma-Aldrich) overnight at $4^{\circ} \mathrm{C}$. The next day, spirochetes were restained with primary antibody (rabbit anti-B. burgdorferi) diluted 1:200 in 0.1\% Triton in PBS (PBS-Tx) for $1 \mathrm{~h}$ at room temperature, and washed $3 \times 5 \mathrm{~min}$ with $0.1 \%$ PBS-Tx. Spirochetes were then stained with anti-rabbit secondary antibody (Alexa 488) (Molecular Probes), diluted 1:500 in PBS-Tx for $1 \mathrm{~h}$ at RT. Cells were then washed with PBS-Tx and nuclei were counterstained with DAPI for $10 \mathrm{~min}$. After mounting in DABCO (Sigma), the number of phagocytic hemocytes was counted using a 488/594 (FITC/TexasRed) dual filter and BX51 fluorescent microscope (Olympus). For each sample, 100 hemocytes were counted.

For the experiment with latex beads (LtxB), $138 \mathrm{nl}$ of $2 \mathrm{x}$ diluted surfactant-free red CML latex beads, $0.3 \mu \mathrm{m}$ diameter (Interfacial Dynamics Corp.) were injected into semi-engorged fed females and, after $2 \mathrm{~h}$, ticks were injected with B. afzelii CB43 $\left(5 \times 10^{4}\right)$. Hemolymph from individual ticks was collected $3 \mathrm{~h}$ after injection of Borrelia and phagocytic activity of hemocytes was analyzed (12 ticks for PBS control group and 15 ticks for latex beads group) as described above. The phagocytic index was determined as the number of hemocytes with ingested Borrelia counted for a total 100 hemocytes in the microscopic field.

\section{Determination of Hemocyte Number after Injection of Latex Beads}

Unfed $I$. ricinus females (25 per group) were injected with 138 $\mathrm{nl}$ of $2 \mathrm{x}$ diluted LtxB or PBS (control) and allowed to rest for 1 day. After that, females were fed naturally on guinea pigs for 6 days and then hemolymph samples from individual females were collected on microscope slides. Cells were fixed with $4 \%$ formaldehyde in PBS for $20 \mathrm{~min}$ and washed 3 times with PBS. Hemocyte nuclei were counterstained with DAPI. Hemocytes were counted on the whole slide using a BX51 Olympus fluorescence microscope.

\section{RNAi-Mediated Silencing of Tick TEPs Linked with In vitro Phagocytosis Assay}

dsRNAs of nine t-teps and $g f p$ (control) were produced as described previously (Buresova et al., 2011). For each experiment, t-teps-specific dsRNA $(0.5 \mu \mathrm{l} ; 3 \mu \mathrm{g} / \mu \mathrm{l})$ was injected into the hemocoel of 25 unfed $I$. ricinus females through to the coxae using a microinjector (Drummond). Ticks were allowed to rest for 1 day and then fed for 6 days on guinea pigs. Hemolymph samples collected from 25 semi-engorged females were mixed with L15-BOFES medium supplemented with $10 \%$ fetal calf serum (PAA Laboratories) (Buresova et al., 2011; Urbanova et al., 2015). Hemocytes $\left(4 \times 10^{4}\right)$ in a volume of $150 \mu \mathrm{l}$ were transferred onto round microscope cover slips in a 24well culture plate, and then $10 \mu \mathrm{l}$ of B. afzelii CB43 $\left(10^{8}\right.$ cells $/ \mathrm{ml}$ ) were added and incubated for $2 \mathrm{~h}$ at $28^{\circ} \mathrm{C}$. Cells were fixed with $4 \%$ formaldehyde in PBS for $20 \mathrm{~min}$ and washed 3 times with PBS. Spirochetes were detected by indirect immunofluorescence, using the method of double staining as described above. Phagocytosed spirochetes were counted using the BX51 Olympus fluorescence microscope. For each group, 100 hemocytes were counted on each of at least 14 slides. Relative phagocytosis was calculated in relation to the number of phagocytic hemocytes in the $g f p$ dsRNA injected control group, taken as $100 \%$ for each respective experiment.

\section{Expression of Genes Encoding I. ricinus Thioester-Containing Proteins in Response to Injection of Borrelia sp. Spirochetes}

Different species of Borrelia (B. burgdorferi NE5264, B. burgdorferi CB26, B. garinii MSLB, B. afzelii CB43) were cultivated in BSK-H complete medium (Sigma) at $33^{\circ} \mathrm{C}$ for $5-7$ days. All Borrelia species were diluted in PBS to contain $10^{4}$ spirochetes in the injection dose. Unfed, pathogen free I. ricinus females were surface-sterilized by a subsequent immersion into $3 \% \mathrm{H}_{2} \mathrm{O}_{2}, 70 \% \mathrm{EtOH}$ and sterile distilled water. A $69 \mathrm{nl}$ volume of Borrelia suspension in sterile PBS, or BSK-H medium for controls, was injected into ticks using sterile glass capillaries and a microinjector (Drummond). After inoculation, ticks were allowed to rest for $12 \mathrm{~h}$ at room temperature, total RNA was 
extracted from the whole body homogenates using TRI reagent ${ }^{\circledR}$ (Sigma), treated with DNAse (Ambion), and the integrity of RNA was checked by agarose gel electrophoresis. Single-stranded cDNA was reverse-transcribed from $0.5 \mu \mathrm{g}$ of total RNA using the Transcriptor High-Fidelity cDNA Synthesis Kit (Roche). The resulting $\mathrm{CDNA}$ preparations served as templates for subsequent expression analysis by quantitative real-time PCR (qPCR) using a LightCycler 480 (Roche) and SYBR green chemistry. Reaction conditions and sequences of qPCR T-TEPs forward and reverse primers have been published previously (Urbanova et al., 2015). Relative expression of t-teps was normalized to elongation factor 1 (ef-1) using the mathematical model of Pfaffl (Pfaffl, 2001). For each experimental group, five $I$. ricinus females were injected in three independent biological triplicates.

\section{Borrelia Transmission}

B. afzelii CB43 spirochetes were cultivated as described above. To prepare Borrelia-infected nymphs for the transmission experiment, $\mathrm{C} 3 \mathrm{H} / \mathrm{HeN}$ mice were injected intra-dermally with $10^{5}$ of $B$. afzelii spirochetes. After 4 weeks, pathogen-free larvae were fed on infected mice ( 100 larvae per mouse) and after repletion were kept in wet chambers at $26^{\circ} \mathrm{C}$ until molting. The infected nymphs (50 per group) were injected with mixed or individual dsRNAs $(3 \mu \mathrm{g} / \mu \mathrm{l}, 64.4 \mathrm{nl})$ specifically silencing the group of IrAMs (iram-1,2,3), IrC3s (irc3-1,2,3), IrMCRs (irmcr-1,2), IrTep (irtep), gfp (control). For experiments with latex beads, 50 unfed $B$. afzelii-infected nymphs were microinjected with $\operatorname{LtxB}$ ( $32 \mathrm{nl}, 2 \mathrm{x}$ diluted) and 50 with sterile PBS as a control group. Following injection, nymphs were allowed to rest for 3 days, and then were fed until repletion on clean 6-weeks old $\mathrm{C} 3 \mathrm{H} / \mathrm{HeN}$ mice (10 nymphs per mouse, 5 mice per each experimental group) using plastic cylinders attached to the murine back. Infection of mice with Borrelia during the early phase following tick infestation was tested in ear tissue biopsies taken at 1 week intervals. After 4 weeks the mice were sacrificed and the Borrelia spirochetes were detected in the target tissues, namely the bladder and heart. The mice tissues were first tested for Borrelia positivity using sensitive PCR amplification of a $154 \mathrm{bp}$ fragment of the flagellin gene. The PCR reactions contained $12.5 \mu \mathrm{l}$ of FastStart PCR MasterMix (Roche), $4 \mu \mathrm{l}$ of DNA extracted using Macherey-Nagel NucleoSpin ${ }^{\circledR}$ Tissue Kit (concentration in the range of 100-300 ng), 10 pmol of each primer FlaF1 (AAGCAAATTTAGGTGCTTTCCAA), FlaR1 (GCAATCATTGCCATTGCAGA) and PCR water up to $25 \mu \mathrm{l}$. The amplification program consisted of denaturation at $94^{\circ} \mathrm{C}$ for $10 \mathrm{~min}$, then 40 cycles of: denaturation at $94^{\circ} \mathrm{C}$ for $30 \mathrm{~s}$, annealing at $60^{\circ} \mathrm{C}$ for $30 \mathrm{~s}$ and elongation at $72^{\circ} \mathrm{C}$ for $40 \mathrm{~s}$. The program was finished by final extension at $72^{\circ} \mathrm{C}$ for $7 \mathrm{~min}$. PCR products were visualized on a $1.5 \%$ agarose gel. Positive tissues were further analyzed by qPCR using a LightCycler 480 (Roche). qPCR was performed in a $25 \mu \mathrm{l}$ reaction volume containing $12.5 \mu \mathrm{l}$ of FastStart Universal Probe Master (Rox) (Roche), $5 \mu \mathrm{l}$ of purified DNA, $10 \mathrm{pmol}$ of each primer, FlaF1, FlaR1, and 5 pmol of TaqMan probe, FlaProbe1 (FAMTGCTACAACCTCATCTGTCATTGTAGCATCTTTTATTTGBHQ1) (Schwaiger et al., 2001). The remaining reaction volume was adjusted with PCR water. The qPCR program consisted of denaturation at $95^{\circ} \mathrm{C}$ for $10 \mathrm{~min}$, followed by 50 cycles of: denaturation at $95^{\circ} \mathrm{C}$ for $15 \mathrm{~s}$, annealing plus elongation at $60^{\circ} \mathrm{C}$ for $1 \mathrm{~min}$. The number of spirochetes in tissues was normalized to the number of murine genomes as described previously (Dai et al., 2009).

\section{Statistical Analysis}

The appropriate statistical analyses (non-parametric KruskalWallis test, non-parametric Mann-Whitney test or un-paired $t$ test) were selected for the specific data-sets and specified in the legends to the corresponding figures. All statistics was performed using GraphPadPrism (version 6.00 for Windows, GraphPad Software, San Diego, CA, USA). A $P$-value of $<0.05$ was considered to be statistically significant.

\section{RESULTS}

\section{Ixodes ricinus Plasma Did Not Exhibit Complement-Mediated Borreliacidal Activity Similar to Bovine Serum}

Complement systems in sera of various vertebrate animals exhibit different borreliacidal effects against different Borrelia genospecies (Kurtenbach et al., 2002; Bhide et al., 2005; Ticha et al., 2016). Because Ixodes sp. ticks possess a primordial complement system involving three molecules related to the C3 complement component and putative convertases (Buresova et al., 2011; Kopacek et al., 2012; Urbanova et al., 2014) we tested for possible effects of tick hemolymph on Borrelia viability. Cultivated $B$. afzelii spirochetes were incubated with tick cell-free plasma, bovine serum, heat-inactivated bovine serum or BSK-H medium and tested for Borrelia survival after $24 \mathrm{~h}$ of incubation in vitro. Incubation of bovine serum with $B$. afzelii resulted in spirochete immobilization, lysis and cluster formation. This effect was markedly reduced by heat inactivation of serum complement. In contrast, no borreliacidal effect was observed upon incubation of spirochetes with tick plasma or BSK-H medium used as a negative control (Figure 1).

\section{Tick Hemocytes Were Capable of Phagocytosing Borrelia afzelii Injected into the Hemocoel}

As no humoral reaction against $B$. afzelii was observed in tick plasma, we further investigated phagocytic activity of tick hemocytes against spirochetes injected into the tick hemocoel. B. afzelii spirochetes were injected into semi-engorged females, and phagocytosis was examined in hemolymph collected at different time intervals post injection. In order to distinguish between Borrelia spirochetes that were ingested by tick hemocytes from attached or free spirochetes, a dual-labeling assay was exploited (Figure 2A). We observed that spirochetes were phagocytosed immediately after injection and a phagocytic rate of about $25 \%$ was reached after $1 \mathrm{~h}$; this was maintained for at least $6 \mathrm{~h}$ (Figure 2B). 


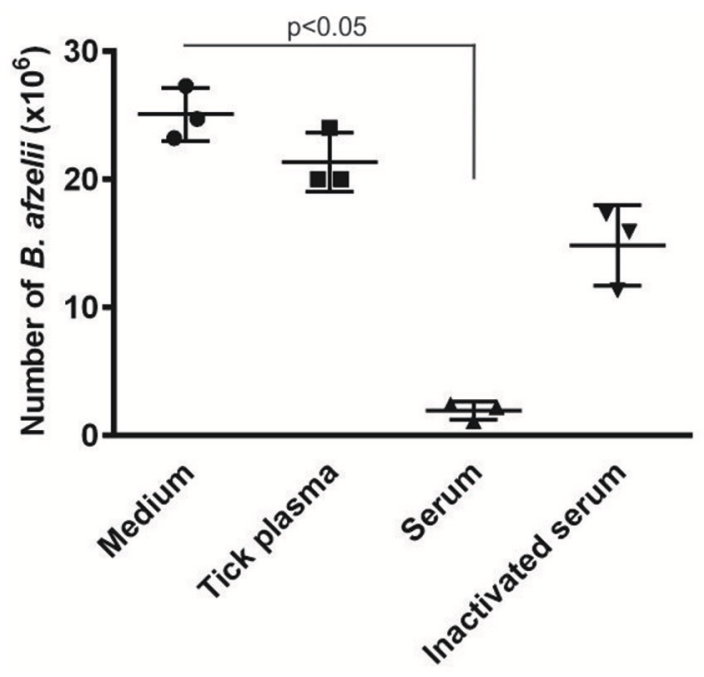

FIGURE 1 | Plasma of $\boldsymbol{l}$. ricinus exhibits no borreliacidal activity against B. afzelii spirochetes. Spirochetes were incubated for $24 \mathrm{~h}$ in the presence of BSK-H medium (negative control), I. ricinus hemocyte-free plasma, bovine serum or heat-inactivated bovine serum. Numbers of live Borrelia were normalized to $1 \mathrm{ml}$ volume. This result demonstrates that complement-like molecules in tick hemolymph do not exert borreliacidal effects similar to that of the complement system present in bovine serum. Data were analyzed by non-parametric Kruskal-Wallis test.

\section{Phagocytosis of $B$. afzelii Was Eliminated by Injection of Latex Beads}

Following the evidence that injection of latex or polystyrene beads suppresses phagocytosis in fruit fly (Nehme et al., 2011) or ticks (Liu et al., 2011), we performed an experiment whereby LtxB was injected into the hemocoel of semi-engorged $I$. ricinus females $2 \mathrm{~h}$ prior to injection of $B$. afzelii. Phagocytosis was evaluated as described above, $3 \mathrm{~h}$ after spirochete injection (Figure 3A). Pre-injection of LtxB resulted in a significant reduction in spirochete phagocytosis, where the phagocytic index decreased from $27 \%$ for the PBS control to $4 \%$ for LtxB preinjection, while the numbers of hemocytes $5 \mathrm{~h}$ after LtxB or PBS injections were the same (Figure 3B).

Intriguingly, injection of LtxB into the hemocoel of unfed females that were further allowed to feed naturally for 6 days resulted in almost complete clearance of hemocytes from tick hemolymph compared to ticks pre-injected with PBS as a control (Figure 4). Despite this striking phenotype, the ticks were capable of feeding with no obvious impact on their fitness or fecundity.

\section{Function of Tick TEPs in Phagocytosis of B. afzelii}

In previous work, we demonstrated that different T-TEPs play non-redundant roles in the phagocytosis of Gram-negative bacteria (Buresova et al., 2011) or the yeast C. albicans (Urbanova et al., 2015). A similar experimental setup combining RNAimediated silencing of individual T-TEPs followed by an in vitro phagocytosis assay was used to identify T-TEPs playing a role in the phagocytosis of Borrelia spirochetes by tick hemocytes. Unfed $I$. ricinus females were injected with gene-specific $t$-teps dsRNA or $g f p$ dsRNA as a negative control. Ticks were allowed to feed naturally for 6 days, then the hemolymph was collected from semi-engorged females and used for an in vitro phagocytosis assay based on $B$. afzelii double immunostaining. Out of nine T-TEPs tested, only silencing of $i r c 3-2$ and irc3-3 significantly decreased phagocytosis of $B$. afzelii. In contrast, knockdown (KD) of the insect-type irtep led to a surprising increase in phagocytosis of spirochetes, by about $20 \%$ compared to the GFP control (Figure 5).

\section{Expression Response of Genes Encoding I. ricinus Thioester-Containing Proteins to Injection of Borrelia sp. Spirochetes}

We have previously shown that expression of genes encoding I. ricinus $\mathrm{T}$-TEPs responds differentially to different model microbes (E. coli, Micrococcus luteus, or C. albicans) or to aseptic injury (Urbanova et al., 2015). Here we have analyzed the expression response of $t$-teps to injection of available species of the $B$. burgdorferi sensu lato complex. Surface-sterilized unfed I. ricinus females were injected with sterile PBS as an aseptic injection control (injury), four different cultivated Borrelia species, and BSK-H medium alone as a mock. Total RNA was isolated $12 \mathrm{~h}$ after injection from whole body homogenates and mRNA levels of the genes encoding t-teps were determined by qPCR. Gene irc3-1 was the only t-tep for which, expression seemed to be up-regulated upon injection of the tested Borrelia genospecies (2-3 times, in relation to the PBS and BSK-H injection controls) (Figure 6). Expression of other t-teps did not change in response to any Borrelia species or injection injury (Figure S1).

\section{Changes in Borrelia Phagocytosis in Ticks Had No Effect on Spirochete Burden in Murine Tissues}

In order to examine whether or not immune reactions in the tick hemocoel affect Borrelia transmission to the host, we adapted the laboratory model for Borrelia transmission developed for B. burgdorferi sensu stricto and I. scapularis (Ramamoorthi et al., 2005; Dai et al., 2009) and applied it for I. ricinus nymphs infected with $B$. afzelii $\mathrm{CB} 43$ as described above. Groups of unfed, infected nymphs were injected with four combinations of dsRNAs, corresponding to the four classes of tick TEPs: (i) $\alpha_{2}$-macroglobulins ( $\operatorname{IrA}_{2} \mathrm{M}-1,2,3$ ); (ii) C3-complement component (IrC3-1,2,3); (iii) macroglobulincomplement-related (IrMcr-1,2), and (iv) insect-type IrTep, respectively. The efficiency of RNAi combinatorial KD was verified by qRT-PCR using CDNA prepared from a pool of five randomly selected nymphs. Additionally, we also tested whether the elimination of phagocytosis that followed pre-injection of LtxB (Figure 3B) affected transmission of B. afzelii. Mice infected with Borrelia during the early phase following tick infestation were tested in ear tissue biopsies taken at 1 week intervals. After 4 weeks, the mice were sacrificed and Borrelia spirochetes were detected in the bladder and heart (Table 1). The Borrelia numbers 


\section{A}

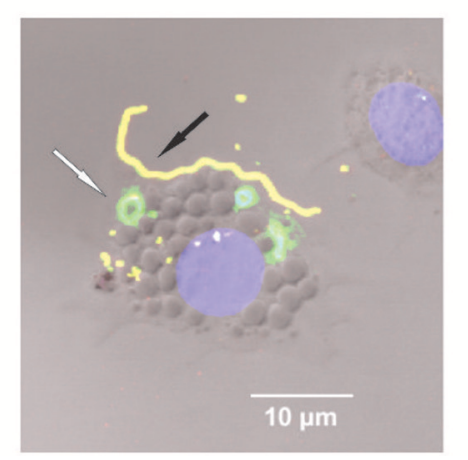

B

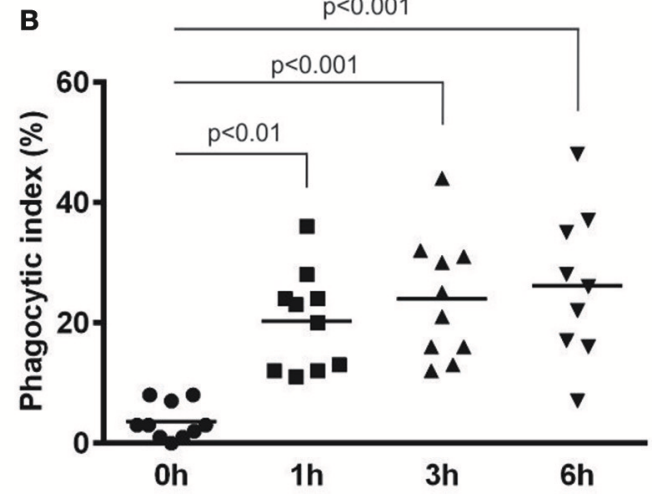

FIGURE 2 | B. afzelii spirochetes are actively phagocytosed by tick hemocytes upon inoculation into the tick hemocoel. (A) Double immunostaining of phagocytic I. ricinus hemocytes distinguished between ingested and attached B. afzelii. Green-ingested Borrelia inside the hemocyte (white arrow); Yellow - attached Borrelia outside the hemocyte (black arrow); Blue-nucleus of hemocyte-stained with DAPI; Nomarski contrast. (B) Semi-engorged females were injected with $5 \times$ $10^{4}$ of $B$. afzelii CB43. Hemolymph samples from different time points after injection were collected from individual ticks (10 ticks per time point) and the phagocytic index was determined as the number of hemocytes with ingested Borrelia per 100 hemocytes in the microscope field. Data were analyzed by non-parametric Kruskal-Wallis test.

in ear biopsies fluctuated, ranging from a few to several thousand spirochetes, usually reaching a maxima from the 2 nd to 3 rd week following infestation and then gradually decreasing (Table S1). The spirochete burdens in bladders and hearts in the 4 th week after infestation were relatively stable, in the range of several hundreds of spirochetes per murine genome (Figure 7). The only statistically significant decrease of Borrelia load compared to the GFP control was observed in mice heart upon group silencing of irc3-1,2,3 genes. Based on these results we conclude that T-TEPS and phagocytosis do not substantially affect the competence of $I$. ricinus to act as a vector for Lyme disease.

\section{DISCUSSION}

The concept of transmission of Lyme disease spirochetes from infected Ixodes sp. ticks to susceptible hosts via the salivary route, as proposed in the late eighties (Ribeiro et al., 1987), has been corroborated by a number of seminal studies published over the past three decades (De Silva and Fikrig, 1995; Coleman et al., 1997; Hojgaard et al., 2008; Dunham-Ems et al., 2009). Several tick proteins have been demonstrated to be involved in tickBorrelia interactions and play roles in spirochete acquisition, midgut colonization, penetration of the midgut epithelium or shielding Borrelia against host immune and inflammatory responses at the tick-host interface (Pal et al., 2004; Ramamoorthi et al., 2005; Narasimhan et al., 2007; Dai et al., 2010; Schuijt et al., 2011; Zhang et al., 2011) or see (Hajdusek et al., 2013; Kung et al., 2013) for review. Except for the recently described antimicrobial peptide Dae2 (domesticated amidase effector), induced by tick GTPase (Chou et al., 2015; Smith et al., 2016), no other tick molecule has been described to limit Borrelia proliferation within a tick vector.

We have previously reported that ticks possess molecules related to components of the mammalian complement system (Buresova et al., 2011; Kopacek et al., 2012; Urbanova et al.,
2015) and therefore we questioned whether tick cell-free plasma can exhibit lytic activity against Borrelia, as demonstrated for a variety of vertebrate animals (Kurtenbach et al., 1998, 2002; Kuo et al., 2000; Bhide et al., 2005; De Taeye et al., 2013; Ticha et al., 2016). The results shown in the Figure $\mathbf{1}$ clearly demonstrate that there is almost no effect of I. ricinus plasma against cultivated spirochetes compared to bovine serum, which is known to have strong complement-mediated borreliacidal activity (Kurtenbach et al., 1998; Bhide et al., 2005). This result is in accord with previous work demonstrating that plasma from I. scapularis, (the competent Lyme disease vector in the USA) had no borreliacidal activity (Johns et al., 2001). In contrast, plasma from the refractory $D$. variabilis substantially reduced the survival of incubated Borrelia. In addition, the authors also reported that clearance of spirochetes inoculated into the hemocoel of both species was much faster in D. variabilis compared to I. scapularis (Johns et al., 2001).

Among cellular reactions, phagocytosis is believed to play the most important defense role against microbial infections of arthropods, including ticks, and have been reported to suppress Borrelia numbers migrating through the tick hemolymph toward the salivary glands (Munderloh and Kurtti, 1995; Coleman et al., 1997; Dunham-Ems et al., 2009). Two different mechanisms, namely conventional and coiling phagocytosis, were reported for engulfment of $B$. burgdorferi by $I$. ricinus hemocytes (plasmatocytes and granulocytes of type II) similar to that of mammalian phagocytic cells (Rittig et al., 1996). Upon direct inoculation of cultivated B. afzelii into the hemocoel of I. ricinus, we observed an immediate and potent phagocytic activity of tick hemocytes, reaching a phagocytic index of about 25\% (Figure 2). The phagocytic activity of tick hemocytes could be almost completely abolished by pre-injection of latex beads into the hemocoel (Figure 3).

Our in vitro phagocytosis assays following specific gene knockdown of individual $t$-teps demonstrated that phagocytosis 
A

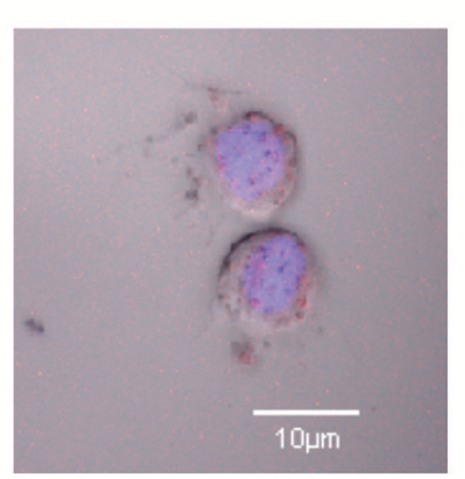

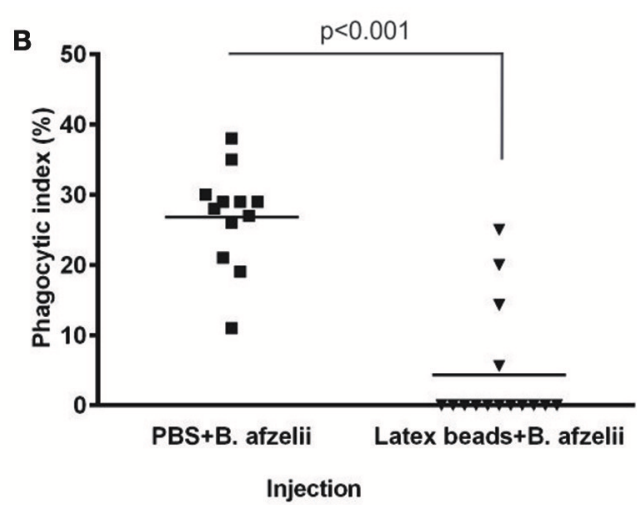

$\mathrm{p}<0.05$

FIGURE 3 | Phagocytosis of $\boldsymbol{B}$. afzelii by tick hemocytes is eliminated by pre-injection of latex beads into the hemocoel. (A) Tick hemocyte with ingested latex beads. Red-latex beads; Blue-nucleus of hemocytes-stained with DAPI; Nomarski contrast. (B) Pre-injection of latex beads (138 nl, $2 x$ diluted) into the hemocoel significantly reduced phagocytosis of $B$. afzelii CB43 injected $2 \mathrm{~h}$ later. Hemolymph was collected from individual ticks (12 ticks for PBS and 15 ticks for latex beads) $3 \mathrm{~h}$ after injection of Borrelia and hemocytes were examined for phagocytosis of spirochetes. The phagocytic index was determined as the number of hemocytes with ingested Borrelia per 100 hemocytes in the microscope field. The number of hemocytes did not differ between experimental groups. Data were analyzed by non-parametric Mann-Whitney test.

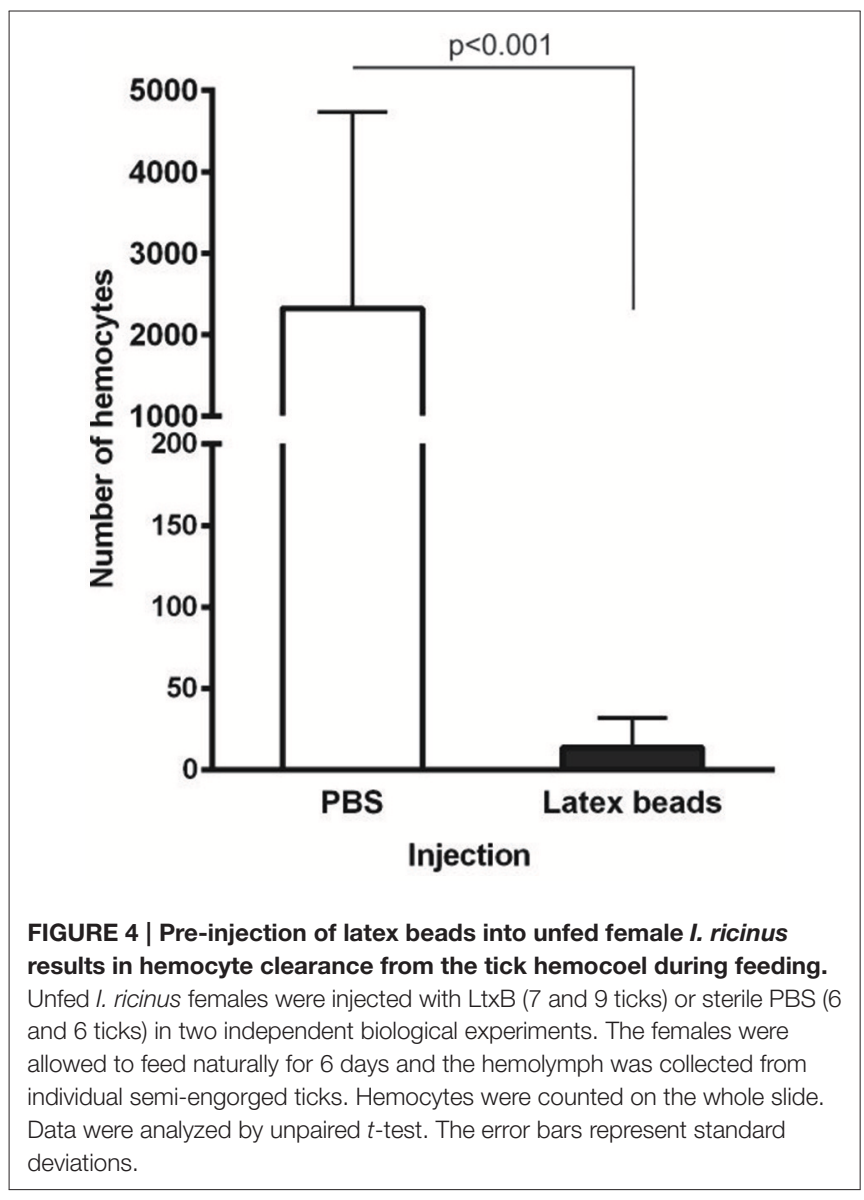

of B. afzelii $\mathrm{CB} 43$ was significantly reduced upon silencing of irc3-2 and irc3-3 and increased upon knockdown of irtep (Figure 5). For comparison, phagocytosis of Gram-negative

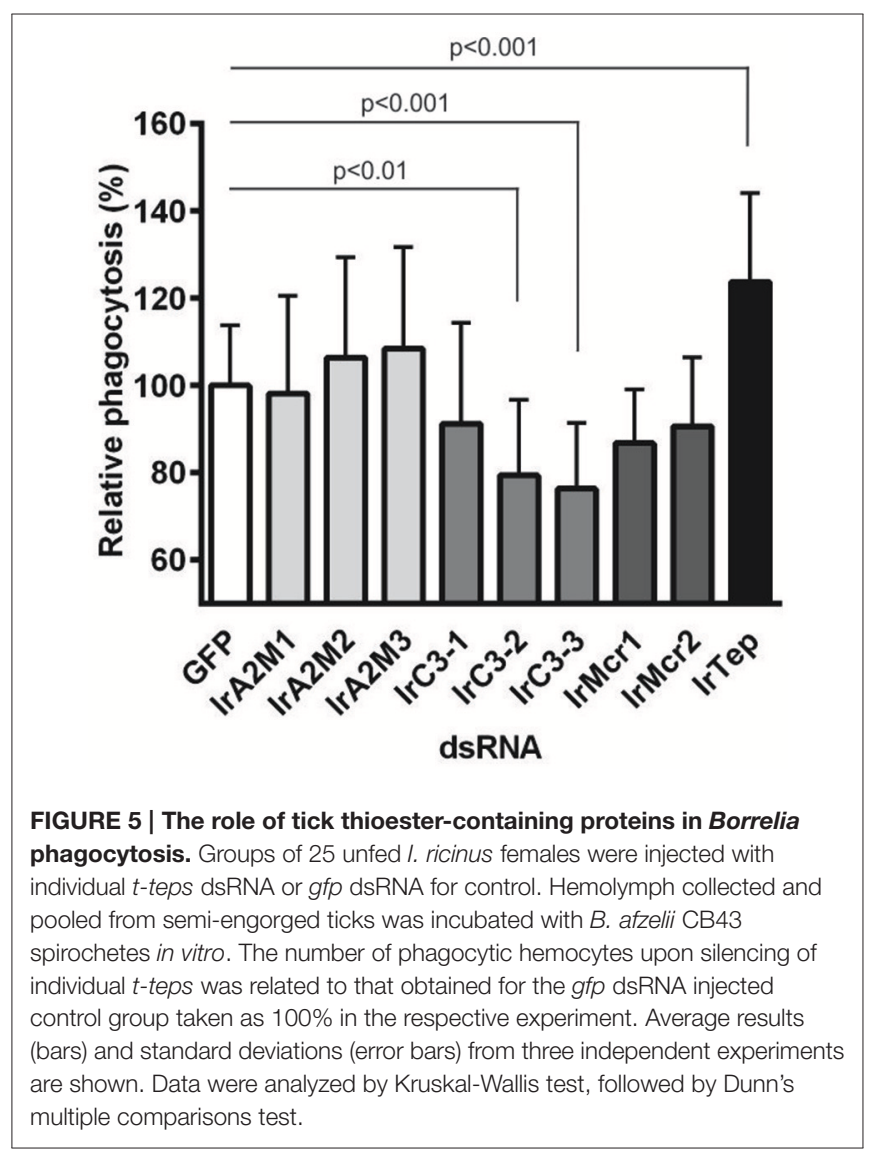

bacteria C. indologenes (pathogenic to ticks) (Buresova et al., 2006) is mediated mainly by $\operatorname{IrA} 2 \mathrm{M}-1,2$ and $\operatorname{IrC} 3-3$. We proposed that involvement of $\alpha_{2}$-macroglobulins in the cellular response against these bacteria was possible given the interaction 
of these macromolecular protease inhibitors with the potent $\mathrm{Zn}^{2+}$-dependent metalloprotease secreted by $C$. indologenes (Buresova et al., 2009). Phagocytosis of the model Gram-negative bacteria E. coli involves IrTep and IrC3-3 (Buresova et al., 2011), whereas for phagocytosis of the yeast C. albicans, the main role is carried out by $\mathrm{IrC} 3-1$ and IrMcr-2 (Urbanova et al., 2015). A similar non-redundant role of members of the TEP family has also been shown for the mosquito Anopheles gambiae, where silencing of $A g$ Tep 1 and $A g$ Tep4 significantly inhibited phagocytosis of $E$. coli as well as the Gram-positive $S$. aureus, while RNAi silencing of mosquito $A g$ Tep3 only reduced phagocytosis of E. coli but not S. aureus (Moita et al., 2005). A study exploiting D. melanogaster S2 cells revealed that DmTep2, $D m$ Tep3 and DmTep6 were specifically required for phagocytosis of E. coli, S. aureus, and C. albicans, respectively (StroscheinStevenson et al., 2006). We also found that inoculation of different strains of the $B$. burgdorferi sensu lato complex upregulated only irc3-1 expression (Figure 6), while expression of other $t$-teps did not seem to be affected (Figure S1). A similar obvious up-regulation of irc3-1 mRNA levels was observed upon injection of C. albicans (Urbanova et al., 2015), suggesting that the immune responses to Borrelia and yeast might be controlled by the same or related signaling pathways.

Earlier, it was elegantly demonstrated using fluorescent (GFPexpressing) B. burgdorferi that penetration of spirochetes from the midgut to the hemocoel was quite a rare event (DunhamEms et al., 2009), which agreed with other studies showing that the number of spirochetes that disseminate in tick hemolymph is very low compared to their massive presence in the tick midgut (Munderloh and Kurtti, 1995; Coleman et al., 1997; Zhang et al., 2011). The fact that the efficient phagocytic response of tick hemocytes is not capable of complete elimination the few Borrelia that migrate through the hemocoel toward the salivary glands could be possibly explained by an extremely fast movement of the motile spirochetes (Malawista and de Boisfleury Chevance, 2008; Dunham-Ems et al., 2009). Assuming that phagocytosis of Borrelia by tick hemocytes indeed reduces their number in the tick hemocoel and thereby negatively affects transmission of the spirochetes to the host via the salivary glands, RNAimediated silencing of $i r c 3-2$ and $i r c 3-3$ should result in a higher Borrelia number in tick salivary glands and subsequently in increased burden in murine tissues. Conversely, stimulation of phagocytosis by silencing of irtep should theoretically lead to reduced infections. However, the T-TEPs group-specific silencing in infected nymphs followed by their feeding on naïve mice did not confirm this view. RNAi-mediated silencing of t-teps did not result in any meaningful relationship with spirochete burden detected in ear biopsies during the early stages of infection. Only group silencing of $\operatorname{irc3-1,2,3}$ resulted in an apparently lower spirochete burden in the murine heart (Figure 7), however, more demanding transmission experiments should be performed either to reinforce or modify the statistical significance of this result for a reasonable interpretation.

Another scenario we can speculate about is that engulfment of spirochetes by tick hemocytes actually protects Borrelia against antimicrobial activity in tick plasma during their movement from the gut to the salivary glands. A "protective" role of

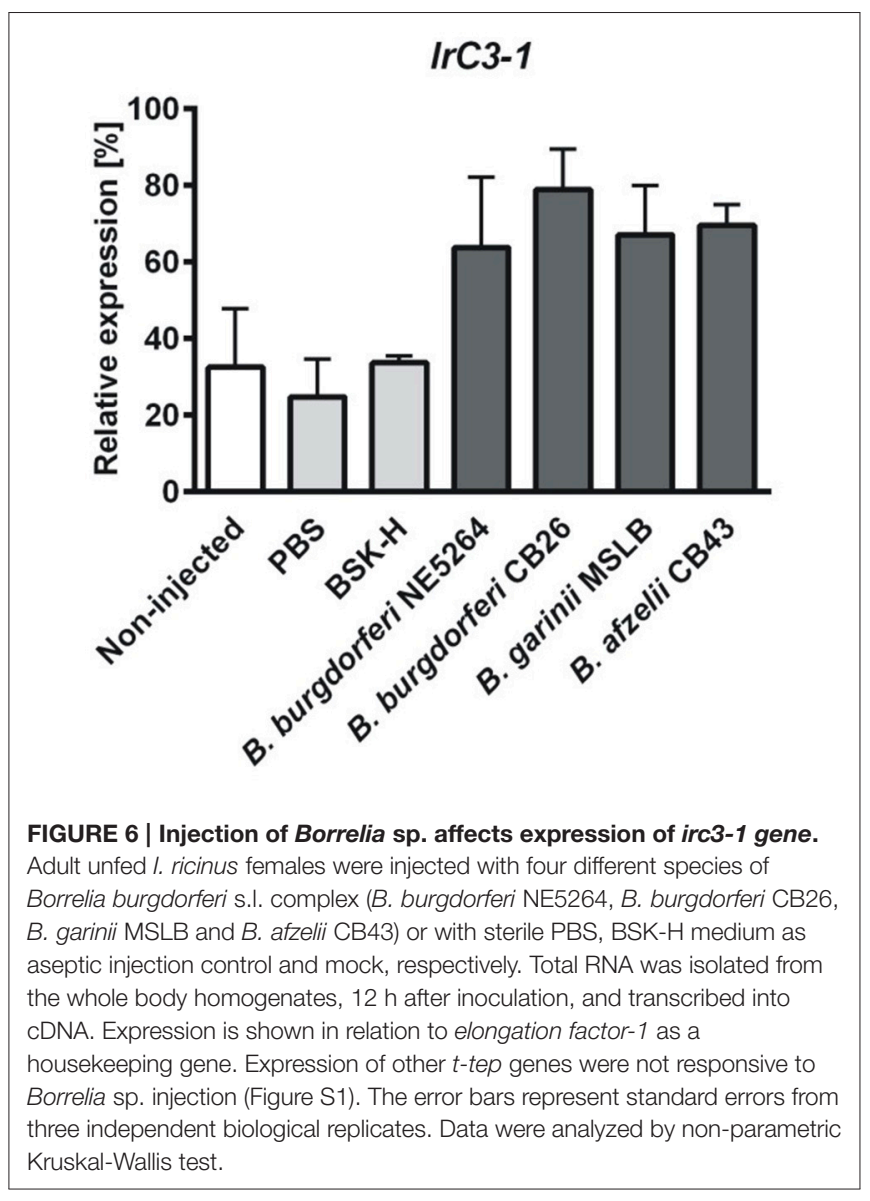

tick hemocytes was proposed for the intracellular tick-borne pathogen, A. phagocytophilum (Liu et al., 2011). Secreted I. scapularis protein 1 (P11) binds to bacteria and facilitates infection of tick hemocytes that serve as a vehicle for the internalized pathogen on its route toward the salivary glands. Silencing of P11 by RNAi or immunization of mice with antiP11 antibodies significantly suppressed the Anaplasma burden in the tick hemolymph or salivary glands. The same effect was achieved by inhibition of $A$. phagocytophilum phagocytosis by injection of polystyrene beads into the I. scapularis hemocoel (Liu et al., 2011). Regarding the extracellular Borrelia spirochetes, the previous work by Johns et al. $(2000,2001)$ reported that $B$. burgdorferi inoculated into the I. scapularis hemocoel were eliminated much more slowly compared to the same experiment performed with the incompetent vector $D$. variabilis. Another in vitro study focused on phagocytosis of GFP-expressing B. burgdorferi by tick cell lines IDE12 and DAE15 derived from $I$. scapularis and D. andersoni, respectively, demonstrated that IDE12 cells required significantly more time to internalize and kill the spirochetes relative to DEA15 cells. Some intact coiled spirochetes (retaining GFP fluorescence) could be found in IDE12 cells as late as 7 days following their co-incubation (Mattila et al., 2007). However, our result showing that preinjection of latex beads into infected nymphs had no apparent effect on Borrelia transmission (Figure 7B) suggests that tick 
TABLE 1 | PCR detection of Borrelia afzelii CB43 ${ }^{a}$ in murine tissues after infestation with infected nymphs pre-injected with dsRNA or latex beads.

\begin{tabular}{|c|c|c|c|c|c|c|}
\hline dsRNA KD/Injection & Ears 1st week & Ears 2nd week & Ears 3rd week & Ears 4th week & Bladder & Heart \\
\hline GFP & $0 / 5$ & $5 / 5$ & $5 / 5$ & $5 / 5$ & $5 / 5$ & $5 / 5$ \\
\hline $\operatorname{Ir} \mathrm{A}_{2} \mathrm{M}-1,2,3$ & $0 / 5$ & $5 / 5$ & $5 / 5$ & $5 / 5$ & $5 / 5$ & $5 / 5$ \\
\hline IrTep & $0 / 5$ & $5 / 5$ & $5 / 5$ & $5 / 5$ & $5 / 5$ & $5 / 5$ \\
\hline IrC3-1,2,3 & $0 / 5$ & $5 / 5$ & $5 / 5$ & $5 / 5$ & $5 / 5$ & $5 / 5$ \\
\hline IrMcr-1,2 & $0 / 5$ & $5 / 5$ & $5 / 5$ & $5 / 5$ & $5 / 5$ & $5 / 5$ \\
\hline Latex beads & $0 / 5$ & $5 / 5$ & $5 / 5$ & $5 / 5$ & $5 / 5$ & $5 / 5$ \\
\hline
\end{tabular}

${ }^{a}$ PCR detection based on amplification of flagellin B gene. Displayed are number of positive/number of examined mice.

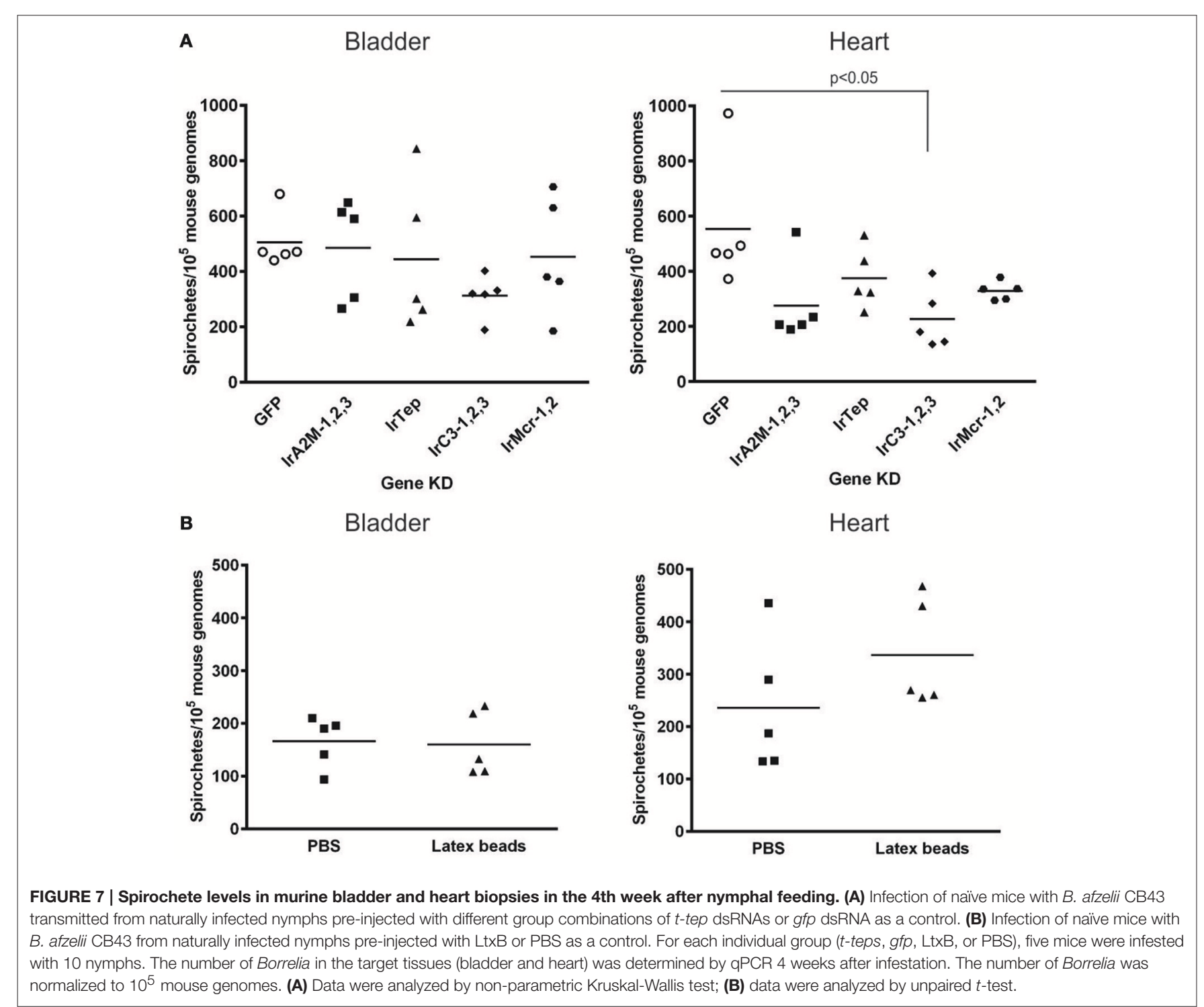

hemocytes do not protect spirochetes on their route toward the salivary glands.

Certainly, many other factors might be affected by manipulating the tick complement-like immune responses, such as an impaired balance between Borrelia spirochetes and the commensal microflora (Narasimhan and Fikrig,
2015), which makes an unequivocal interpretation of our results more difficult. Therefore, more detailed investigations of tick-Borrelia inter-relationships in the midgut, hemocoel and salivary glands of refractory vs. competent tick species (Johns et al., 2001; Soares et al., 2006; Mattila et al., 2007) might shed more light which tissue plays the decisive role 
that determines the tick's capacity to act as a vector for Lyme disease.

\section{AUTHOR CONTRIBUTIONS}

VU, OH, RS, PK conceived the study and designed experiments. $\mathrm{VU}, \mathrm{OH}, \mathrm{HH}, \mathrm{RS}$ performed the experiments and analyzed data. VU, PK wrote the paper.

\section{ACKNOWLEDGMENTS}

This work was primarily supported by the Czech Science Foundation, grant No. $15-12006 \mathrm{Y}$ to VU, additionally by

\section{REFERENCES}

Bhide, M. R., Travnicek, M., Levkutova, M., Curlik, J., Revajova, V., and Levkut, M. (2005). Sensitivity of Borrelia genospecies to serum complement from different animals and human: a host-pathogen relationship. FEMS Immunol. Med. Microbiol. 43, 165-172. doi: 10.1016/j.femsim.2004.07.012

Buresova, V., Franta, Z., and Kopacek, P. (2006). A comparison of Chryseobacterium indologenes pathogenicity to the soft tick Ornithodoros moubata and hard tick Ixodes ricinus. J. Invertebr. Pathol. 93, 96-104. doi: 10.1016/j.jip.2006.05.006

Buresova, V., Hajdusek, O., Franta, Z., Loosova, G., Grunclova, L., Levashina, E. A., et al. (2011). Functional genomics of tick thioester-containing proteins reveal the ancient origin of the complement system. J. Innate Immun. 3, 623-630. doi: $10.1159 / 000328851$

Buresova, V., Hajdusek, O., Franta, Z., Sojka, D., and Kopacek, P. (2009). IrAMAn $\alpha 2$-macroglobulin from the hard tick Ixodes ricinus: characterization and function in phagocytosis of a potential pathogen Chryseobacterium indologenes. Dev. Comp. Immunol. 33, 489-498. doi: 10.1016/j.dci.2008.09.011

Burgdorfer, W., Barbour, A. G., Hayes, S. F., Benach, J. L., Grunwaldt, E., and Davis, J. P. (1982). Lyme disease-a tick-borne spirochetosis? Science 216, 1317-1319.

Chou, S., Daugherty, M. D., Peterson, S. B., Biboy, J., Yang, Y., Jutras, B. L., et al. (2015). Transferred interbacterial antagonism genes augment eukaryotic innate immune function. Nature 518, 98-101. doi: 10.1038/nature13965

Coleman, J. L., Gebbia, J. A., Piesman, J., Degen, J. L., Bugge, T. H., and Benach, J. L. (1997). Plasminogen is required for efficient dissemination of B. burgdorferi in ticks and for enhancement of spirochetemia in mice. Cell 89, 1111-1119.

Dai, J., Narasimhan, S., Zhang, L., Liu, L., Wang, P., and Fikrig, E. (2010). Tick histamine release factor is critical for Ixodes scapularis engorgement and transmission of the lyme disease agent. PLoS Pathog. 6:e1001205. doi: 10.1371/journal.ppat.1001205

Dai, J., Wang, P., Adusumilli, S., Booth, C. J., Narasimhan, S., Anguita, J., et al. (2009). Antibodies against a tick protein, Salp15, protect mice from the Lyme disease agent. Cell Host Microbe 6, 482-492. doi: 10.1016/j.chom.2009.10.006

de la Fuente, J., Estrada-Pena, A., Venzal, J. M., Kocan, K. M., and Sonenshine, D. E. (2008). Overview: ticks as vectors of pathogens that cause disease in humans and animals. Front. Biosci. 13:6938-6946. doi: 10.2741/3200

De Silva, A. M., and Fikrig, E. (1995). Growth and migration of Borrelia burgdorferi in Ixodes ticks during blood feeding. Am. J. Trop. Med. Hyg. 53, 397-404.

De Taeye, S. W., Kreuk, L., Van Dam, A. P., Hovius, J. W., and Schuijt, T. J. (2013). Complement evasion by Borrelia burgdorferi: it takes three to tango. Trends Parasitol. 29, 119-128. doi: 10.1016/j.pt.2012.12.001

Dunham-Ems, S. M., Caimano, M. J., Pal, U., Wolgemuth, C. W., Eggers, C. H., Balic, A., et al. (2009). Live imaging reveals a biphasic mode of dissemination of Borrelia burgdorferi within ticks. J. Clin. Invest. 119, 3652-3665. doi: 10.1172/JCI39401

Hajdusek, O., Sima, R., Ayllon, N., Jalovecka, M., Perner, J., de la Fuente, J., et al. (2013). Interaction of the tick immune system with transmitted pathogens. Front. Cell. Infect. Microbiol. 3:26. doi: 10.3389/fcimb.2013.00026

Hojgaard, A., Eisen, R. J., and Piesman, J. (2008). Transmission dynamics of Borrelia burgdorferi s.s. during the key third day of feeding by nymphal Ixodes grant Nos. 13-11043S, 17-27386S, 17-27393S to $\mathrm{PK}, \mathrm{OH}$, RS, respectively, and by the European Union FP7 project Antidote (Grant Agreement number 602272). The research at the Institute of Parasitology, BC CAS is covered by RVO 60077344. We acknowledge the excellent technical assistance of Gabriela Loosová, Adéla Palusová (Harcubová), and Jan Erhart.

\section{SUPPLEMENTARY MATERIAL}

The Supplementary Material for this article can be found online at: http://journal.frontiersin.org/article/10.3389/fcimb. 2017.00073/full\#supplementary-material

scapularis (Acari: Ixodidae). J. Med. Entomol. 45, 732-736. doi: 10.1603/00222585(2008)45[732:TDOBBS]2.0.CO;2

Johns, R., Ohnishi, J., Broadwater, A., Sonenshine, D. E., De Silva, A. M., and Hynes, W. L. (2001). Contrasts in tick innate immune responses to Borrelia burgdorferi challenge: immunotolerance in Ixodes scapularis versus immunocompetence in Dermacentor variabilis (Acari: Ixodidae). J. Med. Entomol. 38, 99-107. doi: 10.1603/0022-2585-38.1.99

Johns, R., Sonenshine, D. E., and Hynes, W. L. (2000). Response of the tick Dermacentor variabilis (Acari: Ixodidae) to hemocoelic inoculation of Borrelia burgdorferi (Spirochetales). J. Med. Entomol. 37, 265-270. doi: 10.1603/0022-2585-37.2.265

Jongejan, F., and Uilenberg, G. (2004). The global importance of ticks. Parasitology 129(Suppl.), S3-S14. doi: 10.1017/S0031182004005967

Kopacek, P., Hajdusek, O., and Buresova, V. (2012). Tick as a model for the study of a primitive complement system. Adv. Exp. Med. Biol. 710, 83-93. doi: 10.1007/978-1-4419-5638-5_9

Kung, F., Anguita, J., and Pal, U. (2013). Borrelia burgdorferi and tick proteins supporting pathogen persistence in the vector. Future Microbiol. 8, 41-56. doi: $10.2217 /$ fmb.12.121

Kuo, M. M., Lane, R. S., and Giclas, P. C. (2000). A comparative study of mammalian and reptilian alternative pathway of complement-mediated killing of the Lyme disease spirochete (Borrelia burgdorferi). J. Parasitol. 86, 1223-1228. doi: 10.1645/0022-3395(2000)086[1223:ACSOMA]2.0.CO;2

Kurtenbach, K., De Michelis, S., Etti, S., Schafer, S. M., Sewell, H. S., Brade, V., et al. (2002). Host association of Borrelia burgdorferi sensu lato-the key role of host complement. Trends Microbiol 10, 74-79.

Kurtenbach, K., Sewell, H. S., Ogden, N. H., Randolph, S. E., and Nuttall, P. A. (1998). Serum complement sensitivity as a key factor in Lyme disease ecology. Infect Immun. 66, 1248-1251. doi: 10.1016/S0966-842X(01) 02298-3

Liu, L., Narasimhan, S., Dai, J., Zhang, L., Cheng, G., and Fikrig, E. (2011). Ixodes scapularis salivary gland protein P11 facilitates migration of Anaplasma phagocytophilum from the tick gut to salivary glands. EMBO Rep. 12, 1196-1203. doi: 10.1038/embor.2011.177

Malawista, S. E., and de Boisfleury Chevance, A. (2008). Clocking the Lyme spirochete. PLoS ONE 3:e1633. doi: 10.1371/journal.pone.0001633

Mattila, J. T., Munderloh, U. G., and Kurtti, T. J. (2007). Phagocytosis of the Lyme disease spirochete, Borrelia burgdorferi, by cells from the ticks, Ixodes scapularis and Dermacentor andersoni, infected with an endosymbiont, Rickettsia peacockii. J. Insect Sci. 7:58. doi: 10.1673/031.007.5801

Moita, L. F., Wang-Sattler, R., Michel, K., Zimmermann, T., Blandin, S., Levashina, E. A., et al. (2005). In vivo identification of novel regulators and conserved pathways of phagocytosis in A. gambiae. Immunity 23, 65-73. doi: 10.1016/j.immuni.2005. 05.006

Munderloh, U. G., and Kurtti, T. J. (1995). Cellular and molecular interrelationships between ticks and prokaryotic tick-borne pathogens. Annu. Rev. Entomol. 40, 221-243. doi: 10.1146/annurev.en.40.010195. 001253

Narasimhan, S., and Fikrig, E. (2015). Tick microbiome: the force within. Trends Parasitol. 31, 315-323. doi: 10.1016/j.pt.2015.03.010 
Narasimhan, S., Sukumaran, B., Bozdogan, U., Thomas, V., Liang, X., Deponte, K., et al. (2007). A tick antioxidant facilitates the Lyme disease agent's successful migration from the mammalian host to the arthropod vector. Cell Host Microbe 2, 7-18. doi: 10.1016/j.chom.2007.06.001

Nehme, N. T., Quintin, J., Cho, J. H., Lee, J., Lafarge, M. C., Kocks, C., et al. (2011). Relative roles of the cellular and humoral responses in the Drosophila host defense against three gram-positive bacterial infections. PLoS ONE 6:e14743. doi: 10.1371/journal.pone.0014743

Pal, U., Li, X., Wang, T., Montgomery, R. R., Ramamoorthi, N., Desilva, A. M., et al. (2004). TROSPA, an Ixodes scapularis receptor for Borrelia burgdorferi. Cell 119, 457-468. doi: 10.1016/j.cell.2004.10.027

Perner, J., Sobotka, R., Sima, R., Konvickova, J., Sojka, D., Oliveira, P. L., et al. (2016). Acquisition of exogenous haem is essential for tick reproduction. Elife 5:e12318. doi: 10.7554/eLife.12318

Pfaffl, M. W. (2001). A new mathematical model for relative quantification in real-time RT-PCR. Nucleic Acids Res. 29:e45. doi: 10.1093/nar/29.9.e45

Piesman, J., and Gern, L. (2004). Lyme borreliosis in Europe and North America. Parasitology 129(Suppl.), S191-S220. doi: 10.1017/S0031182003004694

Radolf, J. D., Caimano, M. J., Stevenson, B., and Hu, L. T. (2012). Of ticks, mice and men: understanding the dual-host lifestyle of Lyme disease spirochaetes. Nat. Rev. Microbiol. 10, 87-99. doi: 10.1038/nrmicro2714

Ramamoorthi, N., Narasimhan, S., Pal, U., Bao, F., Yang, X. F., Fish, D., et al. (2005). The Lyme disease agent exploits a tick protein to infect the mammalian host. Nature 436, 573-577. doi: 10.1038/nature03812

Ribeiro, J. M., Mather, T. N., Piesman, J., and Spielman, A. (1987). Dissemination and salivary delivery of Lyme disease spirochetes in vector ticks (Acari: Ixodidae). J. Med. Entomol. 24, 201-205.

Rittig, M. G., Kuhn, K. H., Dechant, C. A., Gauckler, A., Modolell, M., RicciardiCastagnoli, P., et al. (1996). Phagocytes from both vertebrate and invertebrate species use "coiling" phagocytosis. Dev. Comp. Immunol. 20, 393-406.

Schuijt, T. J., Coumou, J., Narasimhan, S., Dai, J., Deponte, K., Wouters, D., et al. (2011). A tick mannose-binding lectin inhibitor interferes with the vertebrate complement cascade to enhance transmission of the lyme disease agent. Cell Host Microbe 10, 136-146. doi: 10.1016/j.chom.2011.06.010

Schwaiger, M., Peter, O., and Cassinotti, P. (2001). Routine diagnosis of Borrelia burgdorferi (sensu lato) infections using a real-time PCR assay. Clin. Microbiol. Infect 7, 461-469. doi: 10.1046/j.1198-743x.2001.00282.x

Smith, A. A., Navasa, N., Yang, X., Wilder, C. N., Buyuktanir, O., Marques, A., et al. (2016). Cross-species interferon signaling boosts microbicidal activity within the tick vector. Cell Host Microbe 20, 91-98. doi: 10.1016/j.chom.2016.06.001 Soares, C. A., Zeidner, N. S., Beard, C. B., Dolan, M. C., Dietrich, G., and Piesman, J. (2006). Kinetics of Borrelia burgdorferi infection in larvae of refractory and competent tick vectors. J. Med. Entomol. 43, 61-67. doi: 10.1093/jmedent/43.1.61

Stanek, G., Wormser, G. P., Gray, J., and Strle, F. (2012). Lyme borreliosis. Lancet 379, 461-473. doi: 10.1016/S0140-6736(11)60103-7

Stroschein-Stevenson, S. L., Foley, E., O'farrell, P. H., and Johnson, A. D. (2006). Identification of Drosophila gene products required for phagocytosis of Candida albicans. PLoS Biol. 4:e4. doi: 10.1371/journal.pbio.0040004

Ticha, L., Golovchenko, M., Oliver, J. H. Jr., Grubhoffer, L., and Rudenko, N. (2016). Sensitivity of lyme borreliosis spirochetes to serum complement of regular zoo animals: potential reservoir competence of some exotic vertebrates. Vector Borne Zoonotic Dis. 16, 13-19. doi: 10.1089/vbz.2015.1847

Urbanova, V., Hartmann, D., Grunclova, L., Sima, R., Flemming, T., Hajdusek, O., et al. (2014). IrFC - an Ixodes ricinus injury-responsive molecule related to Limulus Factor C. Dev. Comp. Immunol. 46, 439-447. doi: 10.1016/j.dci.2014.05.016

Urbanova, V., Sima, R., Sauman, I., Hajdusek, O., and Kopacek, P. (2015). Thioester-containing proteins of the tick Ixodes ricinus: gene expression, response to microbial challenge and their role in phagocytosis of the yeast Candida albicans. Dev. Comp. Immunol. 48, 55-64. doi: 10.1016/j.dci.2014.09.004

Zhang, L., Zhang, Y., Adusumilli, S., Liu, L., Narasimhan, S., Dai, J., et al. (2011). Molecular interactions that enable movement of the Lyme disease agent from the tick gut into the hemolymph. PLoS Pathog. 7:e1002079. doi: 10.1371/journal.ppat.1002079

Conflict of Interest Statement: The authors declare that the research was conducted in the absence of any commercial or financial relationships that could be construed as a potential conflict of interest.

Copyright (c) 2017 Urbanová, Hajdušek, Hönig Mondeková, Šíma and Kopáček. This is an open-access article distributed under the terms of the Creative Commons Attribution License (CC BY). The use, distribution or reproduction in other forums is permitted, provided the original author(s) or licensor are credited and that the original publication in this journal is cited, in accordance with accepted academic practice. No use, distribution or reproduction is permitted which does not comply with these terms. 